\title{
The Definition of a Panel of Clinical Biomarkers of Homeostasis: A New Promising Tool for Prevention and Treatment of Chronic-Degenerative Diseases
}

\author{
Anna Maria Berghella*, Ida Contasta and Patrizia Pellegrini
}

Istituto di Farmacologia Traslazionale (IFT), Consiglio Nazionale delle Ricerche (CNR), Unità Operativa di Supporto (UOS), L'Aquila-Italy

For the development of new strategies for prevention and treatment of chronic-degenerative diseases a profound knowledge of natural processes that control and resolve inflammation is required.

Inflammation is a very complex response to an injury, infection, or other stimuli, in which many different cells types and secreted factors orchestrate protective immunity, tissue repair, and resolution of tissue damage. The loss of homeostasis, in this tightly controlled self-regulation process, can lead to chronic inflammation and chronicdegenerative diseases.

Acute inflammation limits and resolves tissue damage, but chronic prolongation of the inflammatory state leads to progressive tissue damage [1], that causes the development of the principal chronicdegenerative pathologies as neoplastic, cardiovascular, autoimmune and neurodegenerative diseases.

In order to identify the guidelines to clarify the natural processes that control and resolve inflammation, it is essential to consider that numerous studies are underlining a strong relationship between inflammatory mediators, aging-related processes and chronic pathologies [2-9]. Furthermore, aging research data identify that the origin and the progression of chronic-degenerative pathologies are related to the loss of physiological homeostasis in the control of inflammation, in which it is now assured the existence of a gender dimorphism [10-13].

Our opinion is that the research on ageing gender-specific immunological pathways and related "Biomarkers of Homeostasis" is a very interesting and promising field for the definition of new pathways that could control the complex inflammatory reactions: these pathways are to be researched in the ageing physio-pathological pathways, specific for gender, that lead, from an healthy state, to low-grade and chronic inflammatory states and to chronic-degenerative pathologies [14-19].

Research data, in fact, indicate that during ageing 1) the chronic inflammation is the base for the origin and the progression of chronic-degenerative diseases, 2) the progressive loss of physiologic homeostasis causes the incapacity to preserve health and that 3 ) gender different disease susceptibility is determined from gender-specific immunological pathways, which suffer adverse changes with ageing [17-19].

Thus, the knowledge of gender physio-pathological pathways, that from an healthy state lead to chronic inflammation, is the scientific ratio for studies that would be interested in the development of strategies to control homeostasis and resolve inflammation. The definition of a panel of clinical biomarkers of homeostasis will be the new tool for prevention and treatment of chronic-degenerative diseases. More specifically, the research procedure consists in the individuation of gender-specific biologic molecules (biomarkers), whose variations could quantify our risk of losing the physiological capacity in the control of the inflammation.
These molecules, in order to satisfy the needs of the objective, have to be measurable in a practical and no-invasive way, in all the following stages: a) the normal state of health, b) the transient (acute) inflammatory state and c) the chronic inflammatory state. The scientific rational is that, to be efficient in the prevention of chronic-degenerative diseases, prognostic biomarkers have to be predictive for the following passages: 1) from the health physiologic condition, in which there is an optimal homeostatic balance in the inflammation control, hence no-risk of pathology, 2) to the transient inflammatory state, where the restoration of this balance is still very probable and there is low-risk of pathology, 3) and/or to the chronic inflammatory state, in which this recovery is physiologically very improbable, while it is relevant the risk of progression toward degenerative pathologies.

These biomarkers allow an early diagnosis and personalized therapeutic interventions, permitting a promising change in the clinical practice and in the sanitary system administration. They open to a large class of prevention programs for these pathologies on the healthy population, that are not yet obtainable, thanks to their usefulness in the identification of subjects that are considered in risk of developing chronic pathologies. On these individuals it is, in fact, justified the application of preventive sanitary procedures, saving the actuation where they are not motivated.

Moreover they are prognostic for the patient stratification in clinic/ therapeutic subgroups: they also permit the quantification of the risk/ benefit related to a therapeutic treatment, allowing the development of the personalized medicine, that could lead to a positive transformation of the clinical strategies. In fact, the risk or benefit of the therapy is connectable to the inability or ability of the specific treatment to restore physiological homeostasis, that is underlined by the changes of the individual biomarker type from low or high-risk to no-risk.

For the selection of suitable molecules, another point that we must consider is that the cellular cytokine environment is a determining key for the immune system homeostasis in the control of inflammation. The cytokine are produced by immunological system cells and regulate the transposition of the information between cells, thanks to the activation

*Corresponding author: Anna Maria Berghella, Istituto di Farmacologia Traslazionale (IFT), Consiglio Nazionale delle Ricerche (CNR), Unità Operativa di Supporto (UOS)-Via G. Carducci, 32 - Rotilio Center, 67100 L'Aquila - Italy, Tel: +39 0862 318843; Fax: +39 0862 320750; E-mail: annamaria.berghella@cnr.it

Received November 27, 2015; Accepted November 28, 2015; Published November 30, 2015

Citation: Berghella AM, Contasta I, Pellegrini P (2015) The Definition of a Pane of Clinical Biomarkers of Homeostasis: A New Promising Tool for Prevention and Treatment of Chronic-Degenerative Diseases. J Mol Biomark Diagn 7: e123. doi:10.4172/2155-9929.1000e123

Copyright: $\odot 2015$ Berghella AM, et al.. This is an open-access article distributed under the terms of the Creative Commons Attribution License, which permits unrestricted use, distribution, and reproduction in any medium, provided the original author and source are credited. 
Citation: Berghella AM, Contasta I, Pellegrini P (2015) The Definition of a Panel of Clinical Biomarkers of Homeostasis: A New Promising Tool for Prevention and Treatment of Chronic-Degenerative Diseases. J Mol Biomark Diagn 7: e123. doi:10.4172/2155-9929.1000e123

Page 2 of 3

of membrane receptors. Therefore, the cytokines can be molecules suitable as biomarkers of the immune system homeostasis in the control of inflammation.

Recent studies [14-19] indicate, for the first time, that the homeostatic equilibrium of the immune response is regulated by cytokines that differ between men and women, and that it is attributable to these differences the different gender trend in the 1) immune response, 2) the predisposition to diseases and 3) the therapeutic response, opening to a new area for the translational research at this level.

These data show that the IFN $\gamma$ cytokine regulates the male immune system homeostasis, while the IL6 cytokine regulate the female one [14,17-19]. Furthermore, these studies explain that a different gender susceptibility and clinical course in diseases is strongly influenced by different polarization of T helper (Th) cell subsets (Treg, Th17 and Th9), determined by the interactions of TGF $\beta$, IL6, IFN $\gamma$ and IL4 cytokine pathways which are different between men and women [14,17-19].

For that reason, the balanced functioning of IFNy and IL6 pathways, respectively in men and in women, is the homeostatic biomarker for the ascertainment of the immune system homeostasis, that controls the inflammation and a healthy longevity.

An equally important point is that the interaction between redox and immunological molecules regulate the gender cytokine immunological homeostasis in the control of inflammation. A key involvement of the interactions between the redox and the immune system in the inflammation and ageing process is concretely underlined by the correlation between the redox state, the functioning of the immune cells and the individual longevity [20-24].

Therefore, relevant in this regard is that CD30, a membrane receptor (R) of the immunological cells, is the specific receptor of Thioredoxin 1 (Trx1) on the immune cells [25] ( $\mathrm{T}$ and B cells, monocytes, dendritic cells, NK, eosinophils and granulocytes).

Trx1 is a protein containing selenocisteine that catalyzes the NADPH-dependent reduction of Thioredoxin reductase (TrxR) and several other oxidized cellular proteins. After an oxidative-stress Trx1 gives origin to cellular signals, activating specific transcriptional factors that regulate the decode in the nucleus of the genes that produce substances providing to the defense of the cell against the reactive oxygen species (ROS), that have induced the oxidative stress condition [26-34].

On the other hand, CD30R is a member of the TNF/NGF $\mathrm{R}$ super-family, it is generally defined as a molecule that mediate cell regulation signals. Research results [35,36-39] clarified and underlined the importance of its physiopathologic function: the pathways that are regulated by the interaction between CD30R and soluble (s) CD30 (its soluble component is released in the environment when RCD30 interact whit its ligand $\mathrm{CD} 30 \mathrm{~L}$ ), control the physiologic homeostasis in the immune and in the neurologic system and they regulate the functions of monocytes and dendritic cells, mature and immature, to direct the T-helper cell differentiation in the respective subtypes (Treg, Th1, Th2, Th9 and Th17).

Then, these results clarify that the functional link between Trx1 and CD30 is a very important step in the physiologic homeostasis and it underlines the big potentiality of these elements as a "double biomarker" of the immune system homeostasis to quantify the physiological ability to resolve inflammation.

Male and female cells, in fact, have a different capacity to defend themselves toward the oxidative stress: the activity and production of ROS seems to be regulated differently in men and women, it could be directly influenced by sexual hormones [40] and constitute a new promising area of research [41,42].

Consequently, based on all the above considerations, the new routes, between the complex inflammatory reactions and the strategies to regulate them have to be researched in the natural process of the well regulated negative feedback mechanisms to resolve rapidly and restore homeostasis at infected or damaged sites.

It is our opinion that the field of this research must be the ageing gender-specific immunological pathways, with the aim to identify a panel of clinical "Biomarkers of Homeostasis," whose variations could quantify our risk of losing the physiological capacity in the control of the inflammation.

To this aim, the interaction between redox and immunological molecules, which regulate the gender cytokine immunological homeostasis in the control of inflammation, is certainly one of the most interesting and promising topics.

\section{References}

1. Nathan C, Ding A (2010) Non resolving inflammation. Cell 40: 871-882.

2. Vasto S, Carruba G, Lio D, Colonna-Romano G, Di Bona D, et al. (2009) Inflammation, ageing and cancer. Mech Ageing Dev 130: 40-45.

3. Krabbe KS, Pedersen M, Bruunsgaard H (2004) Inflammatory mediators in the elderly. Exp Gerontol 39: 687-699.

4. Howcroft TK, Campisi J, Louis GB, Smith MT, Wise B, et al. (2013) The role of inflammation in age-related disease. Aging 1: 84-93.

5. Vasto S, Candore G, Balistreri CR, Caruso M, Colonna-Romano G, et al. (2007) Inflammatory networks in ageing, age-related diseases and longevity. Mech Ageing Dev 128: 83-91.

6. Vasto S, Candore G, Nuzzo D, Mocchegiani E, Di Bona D, et al. (2008) Inflammation, genes and zinc in Alzheimer's disease. Brain Res Rev 58: 96105.

7. Candore G, Colonna-Romano G, Balistreri CL, Di Carlo D, Grimaldi MP, et al., (2006) Biology of longevity: role of the innate immune system. Rejuvenation Res 9: 143-148.

8. De Guire V, Robitaille R, Tétreault N, Guérin R, Ménard C, et al. (2013) Circulating miRNAs as sensitive and specific biomarkers for the diagnosis and monitoring of human diseases: Promises and challenges. Clin Biochem 46 846-860.

9. Capri M, Salvioli S, Monti D, Caruso C, Candore G, et al. (2008) Human longevity within an evolutionary perspective: the peculiar paradigm of a postreproductive genetics. Exp Gerontol, 43: 53-60.

10. Blagosklonny MV (2012) Answering the ultimate question "what is the proxima cause of aging?. Aging 4: 861-877.

11. Pietrantonio F, Braud F, Prat V, Perrone F, Pierotti MA, et al. (2013) A review on biomarkers for prediction of treatment outcome in gastric cancer. Anticancer Res 33: 1257-1266.

12. Kavathia N, Alka J, Walston J, Beamer BA, Fedarko NS (2009) Serum markers of apoptosis decrease with age and cancer stage. Aging, 1: 652-666.

13. Osoriov FG, López-Otín C, Freije JMP (2012) NF-kB in premature aging. Aging 4: 726-727.

14. Pellegrini P, Contasta I, Del Beato T, Ciccone F, Berghella AM (2011) Genderspecific cytokine pathways, targets, and biomarkers for the switch from health to adenoma and colorectal cancer. Clin Dev Immunol.

15. Bettelli E, Carrier Y, Gao W, Korn T, Strom TB, et al. (2006) Reciproca developmental pathways for the generation of pathogenic effector $\mathrm{TH} 17$ and regulatory T cells. Nature 441: 235-238.

16. Oukka, (2008) Th17 cells in immunity and autoimmunity. Annals of the Rheumatic Disease. 67: 26-29. 
Citation: Berghella AM, Contasta I, Pellegrini P (2015) The Definition of a Panel of Clinical Biomarkers of Homeostasis: A New Promising Tool for Prevention and Treatment of Chronic-Degenerative Diseases. J Mol Biomark Diagn 7: e123. doi:10.4172/2155-9929.1000e123

17. Berghella AM, Contasta I, Marulli G, D'Innocenzo C, Garofalo F (2014) Ageing gender-specific "Biomarkers of Homeostasis", to protect ourselves against the diseases of the old age. Immunity \& Ageing 11: 3.

18. Berghella AM, Contasta I, Del Beato T, Ciccone F, Pellegrini P, (2012) The discovery of how gender influences age immunological mechanisms in health and disease, and the identification of ageing gender-specific biomarkers, could lead to specifically tailored treatment and ultimately improve therapeutic success rates. Immunity \& Ageing 9: 24.

19. Contasta I, Totaro R, Pellegrini P, Del Beato T, Berghella AM, et al. (2012) A gender-related action of IFN $\beta$-therapy was found in multiple sclerosis. Journal of Traslational Medicine 10: 223.

20. Du L, Bayir H, Lai Y, Zhang X, Kochanek PM, et al. (2004) Innate gender-based proclivity in response to cytotoxicity and programmed cell death pathway. The Journal of Biological Chemistry 27: 38563-38570.

21. Ortona E, Margutti, P, Matarrese P, Franconi F, Malorni W (2008) Redox state cell death and autoimmune diseases: a gender perspective. Autoimmunity Reviews 7: 579-584.

22. Cannizzo ES, Clement CC, Sahu R, Follo C, Santambrogio L (2001) Oxidative stress, inflamm-aging and immunosenescence. J Proteomics 7: 2313-2323.

23. Buffenstein R, Edrey YH, Yang T, J Mele J (2008) The oxidative stress theory of aging: embattled or invincible? Insights from non-traditional model organisms. Age 30: 99-110.

24. Salminen A, Kaarniranta K, Kauppinen A (2012) Inflammaging: disturbed interplay between autophagy and inflammasomes. Aging 4: 166-175.

25. Schwertassek U, Balmer Y, Gutscher M, Weingarten L, Preuss M, et al. (2007) Selective redox regulation of cytokine receptor signaling by extracellular thioredoxin1. Embo J 26: 3086-3097.

26. Becker K, Gromer S, Schirmer RH, Muller S (2000) Thioredoxin reductase as a pathophysiological factor and drug target. Eur J Biochem 267: 6118-6125.

27. Powis G, Kirkpatrick DL, Angulo M, Baker A (1998) Thioredoxin redox control of cell growth and death and the effects of inhibitor. Chem Biol Interact 112: 23-34

28. Hirota K, Matsui M, Murata M, Hirota MK (2000) Nucleoredoxin, glutaredoxin and thioredoxin differentially regulate NF-_B, AP-1, and CREB activation in HEK293 cells. Biochem Biophys Res Comm 274: 177-182.

29. Hirota K, Nakamura H, Arai T, Ishii H, Itoh T, et al. (2000) Geranylgeranylacetone enhances expression of thioredoxin and suppresses ethanol-induced cytotoxicity in cultured hepatocytes. Biochem Biophys Res Comml 275: 825-830.

30. McDonald PP, Cassatella MA, Bald A, Maggi E, Romagnani S, et al. (1995)
CD30 ligation induces Nuclear Factor kappa B activation in human T cell lines. Eur J Immunol 25: 2870-2876.

31. Del Beato T, Berghella AM, Pellegrini P, Adorno D, Casciani CU (1997) The role of the soluble CD30 serum level in colorectal cancer: a possible marker for a patient subset which could benefit from IL-2 biotherapy. Cancer Biother Radiopharm 12: 297-305

32. Pellegrini P, Berghella AM, Contasta I, Adorno D (2003) CD30: not a physiological marker for $\mathrm{TH} 2$ cells but an important costimulator molecule in the regulation of the balance between $\mathrm{TH} 1 / \mathrm{TH} 2$ respons. Transpl Immunol 12 49-61.

33. Pellegrini $P$, Totaro R, Contasta C, Berghella AM, et al. (2005) CD30 antigen and Multiple Sclerosis. CD30: an important costimulator molecule and marker for a regulatory subpopulation of dendritic cells involved in mantaining the physiological balance between $\mathrm{TH} 1 / \mathrm{TH} 2$ immune response and immune tolerance. Neuroimmunomodulation 12: 220-234.

34. Hargreaves PG, Al-Shamkhani AA (2002) Soluble CD30 blocks transmembrane signaling by CD30. Eur J Immunol 32: 163-17.

35. Murray JS, Madri J, Pasqualini T, Bottomly K (1993) Functional CD4 T cell subset interplay in an intact immune system. J Immunol 150: 4270-4276.

36. Kopf M, Le Gros G, Bachmann M, Lamers MC, Bluethmann H, et al. (1993) Disruptionof the murine IL-4 gene blocks TH2 cytokine responses. Nature 362 245-248.

37. TanakaT, Hu-Li J, Seder RA, Fazekas de St.Groth B, Paul WE (1993) Interleukin 4 suppresses interleukin 2 and interferon gamma production by naive $T$ cells stimulated by accessory cell-dependent receptor engagement. Proc Natl Acad Sci 90: 5914-591.

38. Bradley LM, Yoshimoto K, Swain SL (1995) The cytokines IL-4, IFN-gamma, and $\mathrm{IL}-12$ regulate the development of subsets of memory effector helper $\mathrm{T}$ cells in vitro. J Immunol 155: 713-724.

39. Banchereau J (1995) Converging and diverging properties of human interleukin-4 and interleukin-10. Behring Institute Mitteilungen 96: 58-77.

40. Song JJ, LeeYJ (2003) Differential role of glutaredoxin and thioredoxin in metabolic oxidative stress-induced activation of apoptosis signal regulating kinase1. Biochemical Journal 373: 845-853.

41. Gius D, Boter A, Shah S, Curry HA (1999) Intracellular oxidation/reduction status in the regulation of transcription factors NF-B and AP-1. Toxicol Lett 106: 93-106.

42. Halliwell B, Gutteridge JM (1988) Free radicals and antioxidant protection: mechanisms and significance in toxicology and disease. Human Toxicol 7 : 7-13. 